Archives of Clinical and Medical Case Reports

doi: $10.26502 /$ acmcr. 96550048

fortune

Volume 2, Issue 6

Case Report

\title{
Imaging Findings of Solitary Plasmacytoma of the Cranium
}

\section{Shrestha Sukriti, Zhu Li, Zhao Xinxiang*}

Department of Radiology, The Second Affiliated Hospital of Kunming Medical University, P.R China

"Corresponding Author: Dr. Xinxiang Zhao, Department of Radiology, The Second Affiliated Hospital of Kunming Medical University, 374,Dianmian Road, Wuhua District, Yunnan Province 650000, P.R.China, E-mail: $\underline{13888297841 @ 126 . c o m}$

Received: 25 October 2018; Accepted: 05 November 2018; Published: 19 November 2018

\begin{abstract}
Solitary plasmacytoma of bone (SBP) are extremely rare and limited in the English literature. The most common locations in the skull are sphenoid bone, juxtasellar region, petrous apex and cerebral convexity or falx. SBP has higher risk of advancing to Multiple Myeloma within the duration of 2-4 years. Here we report two cases involving the cranium, both the cases initially mis-diagnosed as meningioma and after histopathology report confirmed as plasmacytoma. Further laboratory investigations were made to rule out multiple myeloma and its systemic involvement. We will also further discuss about the CT and MRI features and the most common differential diagnosis of SBP.
\end{abstract}

Keywords: Solitary plasmacytoma; Cranium; Diagnosis; CT; MRI

\section{Introduction}

Solitary plasmacytoma is an early stage malignancy with a clinical course that lies between MGUS (monoclonal gammopathy of undertermined significance) and multiple myeloma (MM) in the spectrum of plasma cell dyscrasia. It has been divided into two types: one that grows within the axial skeleton known as solitary plasmacytoma of bone (SPB) and the other within soft tissue called extramedullary plasmacytoma (EMP). Skull involvement occurs mainly in cranial vault, skull base, dura and orbit [1]. Cranial vault and cranial base lesions are suggested to have higher risk of evolution to MM than dural lesions [1]. SPB is the commonest variety and accounts for 3-5\% of all plasma cell malignancies [2-4]. Here we report two cases involving the cranium, both the cases initially diagnosed as 
meningioma and after histopathology report confirmed as plasmacytoma. Further investigations were done to rule out MM.

\section{Case I}

A 74-year-old male with history of coronary heart disease presented with left temporal headache and hearing unusual voices for more than 20days. Neurological examination didn't reveal any visual, auditory and verbal abnormalities, gait disorders and ataxia.

\subsection{Radiological findings}

CT scan revealed a $1.3 \times 0.8 \times 1.2 \mathrm{~cm}$ round, well-defined and hyperdense extra-axial mass in the left temporal lobe with homogenous enhancement after contrast administration (Figure 1 and 3). On bone CT, the adjacent skull showed bone defect (Figure 2). MRI scan depicted a fusiform lesion in left temporal lobe with adjacent skull involvement. The lesion appeared isointense on both T1 and T2-weighted images (Figure 4). Diffusion-weighted images (DWI) with b-value of 1000 showed no restricted diffusion (Figure 6). After intravenous gadolinium, the mass demonstrated significant homogenous enhancement (Figure 5). The initial imaging diagnosis was Meningioma.

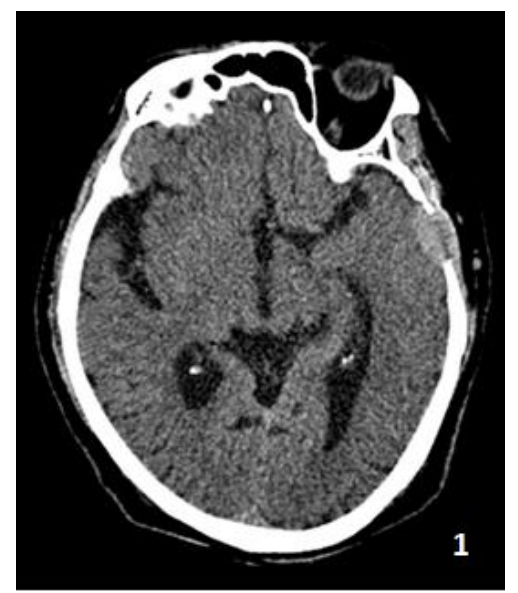

Figure 1: Axial CT showing a hyper dense extra-axial mass in the left temporal lobe.

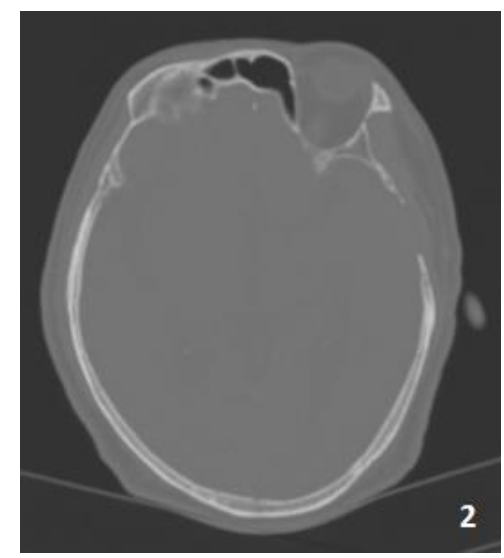

Figure 2: Axial CT bone window showing bone defect in the left temporal lobe. 


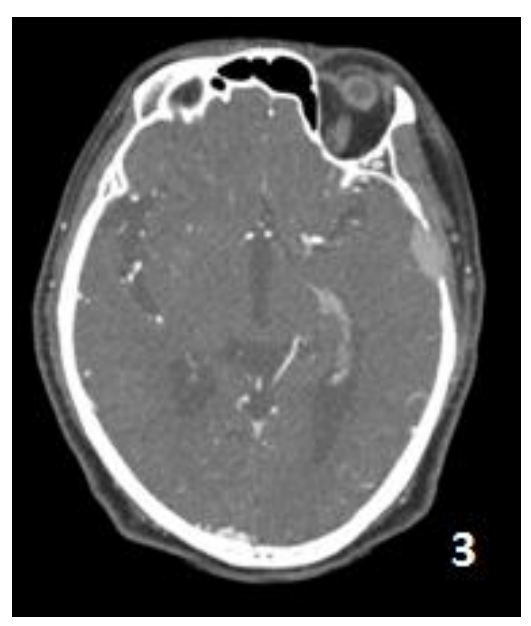

Figure 3: Axial post contrast CT showing homogenous enhancement in the left temporal lobe.

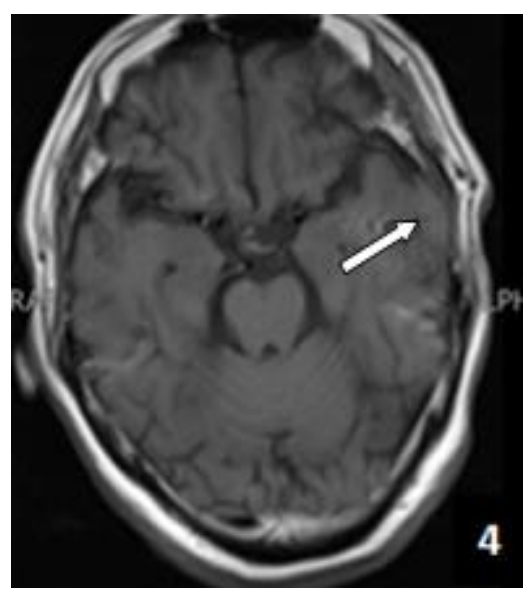

Figure 4: Axial T1 weighted MRI showing a isointense extra-axial mass in the left temporal lobe(Arrow).

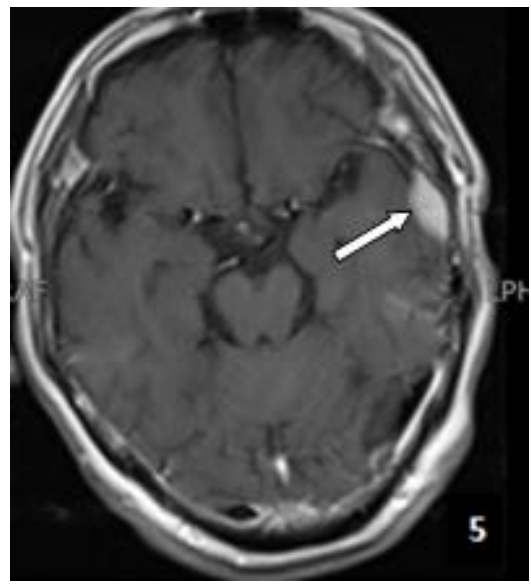

Figure 5: Axial post-contrast MRI showing vivid homogenous enhancement in the left temporal lobe(Arrow). 


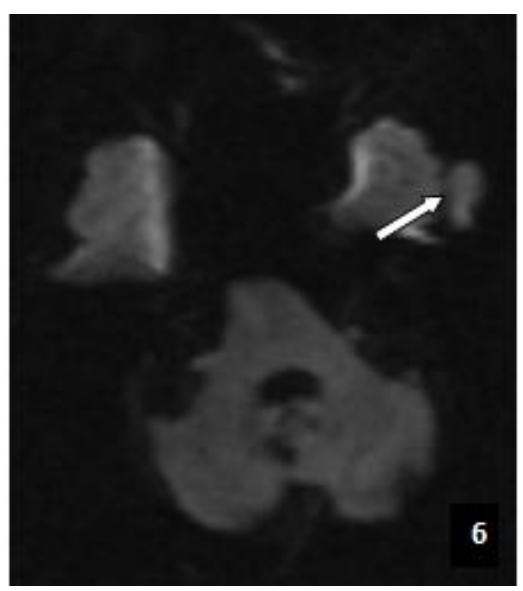

Figure 6: DWI image showing no restricted diffusion(Arrow).

\subsection{Laboratory findings}

His routine blood count showed low lymphocytes (Lymp=16.8\%) and high monocytes (Mono=16.7\%). Red blood cell count $\left(\mathrm{RBC}=4.16 \times 10^{12} / \mathrm{l}\right)$ and hemoglobin $(\mathrm{Hb}=12.9 \mathrm{gm} / \mathrm{l})$ were slightly decreased. Renal function test showed urea $=7.33 \mathrm{mmol} / 1$ and creatinine $(\mathrm{CRE}=70 \mathrm{umol} / \mathrm{l})$. Immunohistochemistry showed embryonal membrane antigen EMA (-), GFAP (-) and CD138 (+) and CD38 (+), k (-), v (-). The pathological diagnosis was plasmacytoma. Then he further underwent investigations to rule out Multiple Myeloma. Bone marrow aspiration revealed $<5 \%$ plasma cells. Bence Jones protein was negative. The serum electrophoresis revealed decreased levels of immunoglobulins: IgG: $4.58 \mathrm{~g} / \mathrm{l}, \mathrm{IgM}: 0.08 \mathrm{~g} / \mathrm{l}, \mathrm{IgA}: 0.14 \mathrm{~g} / \mathrm{l}$. Since none of the criteria's for MM were met, this case was confirmed to be Solitary plasmacytoma of the skull. The patient received postoperative radiotherapy for one year and showed complete remission.

\section{Case II}

A 45-year-old male presented with complains of dizziness lasting for few minutes since more than 6 months. There were no associated symptoms of headache, nausea and vomiting. However, in the past 3 months he had gradual blurring of vision with diplopia. He also developed numbness in his right lower extremity. Neurological examination didn't reveal any verbal and auditory abnormalities.

\subsection{Radiological findings}

CT scan revealed a large, hyperdense extradural mass with mass destruction and skull defect occupying right parietal and occipital lobe (Figure 7 and 8 ). The mass was of size about $5.8 \times 6.5 \times 2.4 \mathrm{~cm}$ with well-defined margin and showed slight enhancement after contrast (Figure 9). MRI revealed isointensity on both T1WI and T2 weighted images (Figure 10). After intravenous gadolinium, the mass showed significant homogenous enhancement with mass effect and right lateral ventricle effacement (Figure 11). Skeletal survey showed primary cancer in right occipital lobe with intensively increased tracer uptake in left $6^{\text {th }}$ rib, left $5^{\text {th }}, 8^{\text {th }}$ and L5 vertebrae. 


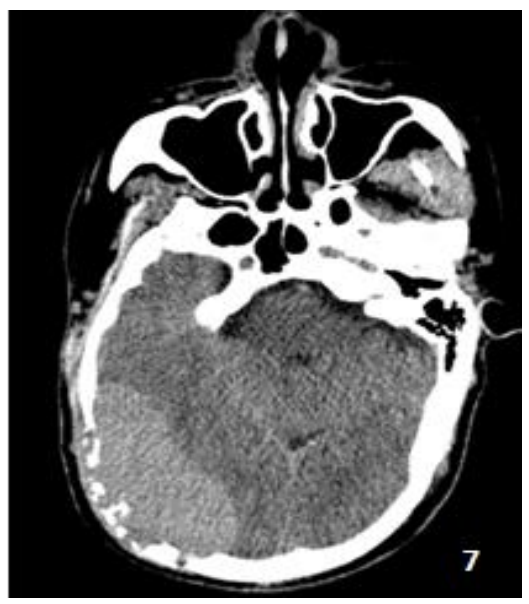

Figure 7: Axial CT showing a large hyperdense mass in right parietal and occipital lobe.

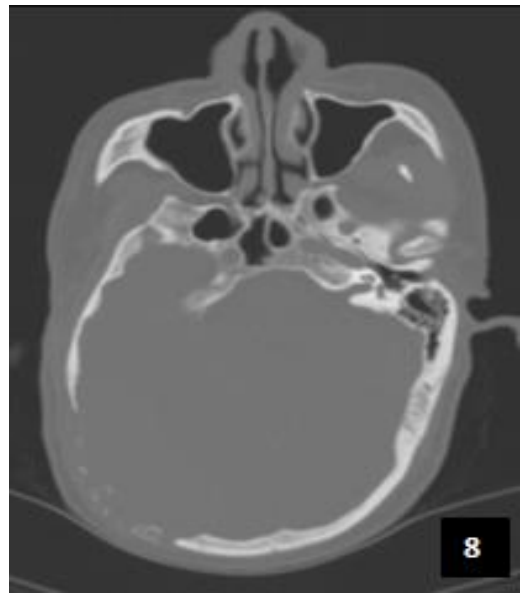

Figure 8: Axial CT-bone showing mass destruction and skull defect in right parietal and occipital lobe.

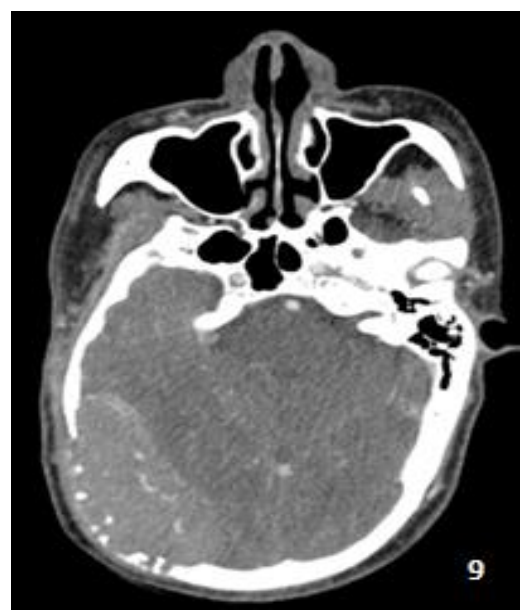

Figure 9: Axial post contrast CT showing homogenous enhancement in right parietal and occipital lobe. 


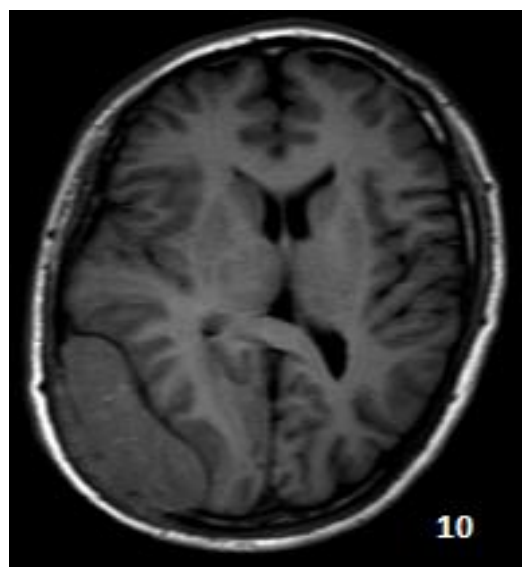

Figure 10: Axial T1-weighted MRI showing a large hyperdense mass in right parietal and occipital lobe.

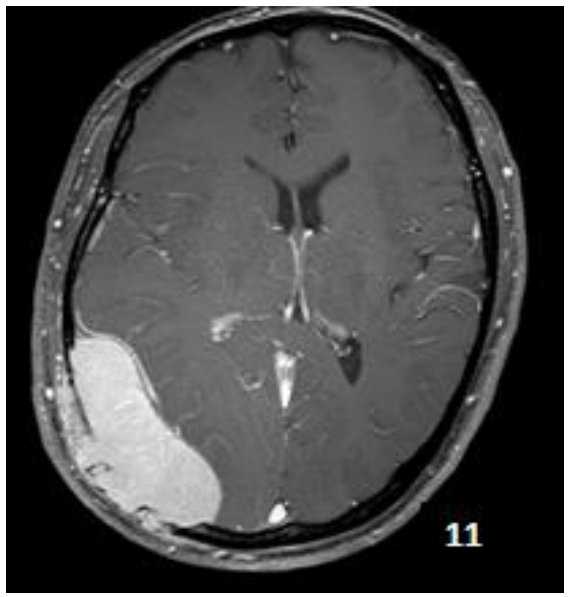

Figure 11: Axial post contrast MRI showing homogenous enhancement in right parietal and occipital lobe.

\subsection{Laboratory findings}

His routine blood count showed low levels of White blood cells $\left(\mathrm{WBC}=0.5 \times 10^{9} / 1\right)$, low neutrophils (Neut=34\%) and high monocytes (Mono=14\%). Red blood cells $\left(\mathrm{RBC}=2.97 \times 10^{12} / \mathrm{l}\right)$, hemoglobin $(\mathrm{Hb}=89 \mathrm{gm} / \mathrm{l})$ and platelets $\left(\mathrm{PLT}=57 \times 10^{9} / \mathrm{l}\right)$ levels were also decreased. Renal function test showed urea $=6.86 \mathrm{mmol} / 1$ and creatinine $(\mathrm{CRE}=80$ umol/1). Immunohistochemistry showed embryonal membrane antigen (EMA) (+), Vimentin (+), CD38 (+), CD138 $(+)$, GFAP (-), k (+), v (+). His pathological report confirmed Plasmacytoma. However, Multiple Myeloma couldn't be ruled out as his serum electrophoresis for M-protein, Bence Jones protein and bone marrow aspiration reports couldn't be traced as the patient didn't follow-up.

\section{Discussion}

Solitary plasmacytoma of bone is rare [2,3]. Incidence rate rises with advancing age; however compared to MM, it is less common at older age. The incidence rate of SP in black race is around 30\% higher than white race [2]. Plasmacytoma is further sub-categorized by International Myeloma Working Group (IMWG) into (i) solitary 
plasmacytoma of bone (SPB); (ii) extramedullary plasmacytoma (EMP), and (iii) multiple plasmacytomas [2,3]. Histologically, plasmacytoma and MM demonstrates cellular resemblance, due to which differentiation of these entities is essential for its management and prognosis. The absence of features of $C R A B$ (increased Calcium, Renal insufficiency, Anemia, or multiple Bone lesions) aids in differentiating myeloma from SBP and EMP [2]. When a plasmacytoma is found along with MM, the patient should be treated for MM [2].

Both SPB and EMP shows male predilection with M: F ratio of 2:1 and 3:1 respectively [3, 4]. The median age is 55 years [2-4]. SPB affects the axial skeleton especially the vertebrae and EMP affects the head and neck, mainly nasal cavity and nasopharynx [2,3]. The median overall survival in different series varies from 7.5 to 12 years $[2,4,5]$. SBP have poor prognosis then EMP [2-4]. Diagnosis of SPB and EMP is made when the recommended criteria of multiple myeloma are absent. The presence of only a single bone lesion, normal bone marrow aspirate ( $<5 \%$ plasma cells), absent or low monoclonal immunoglobulin in serum or urine (level of $>20 \mathrm{~g} / \mathrm{l}$ suspicious of MM), no evidence of organ damage (anemia, hypercalcaemia or renal impairment) and a normal skeletal survey (other than the single bone lesion) confirms SPB [2-4] (Figure 12 and 13).
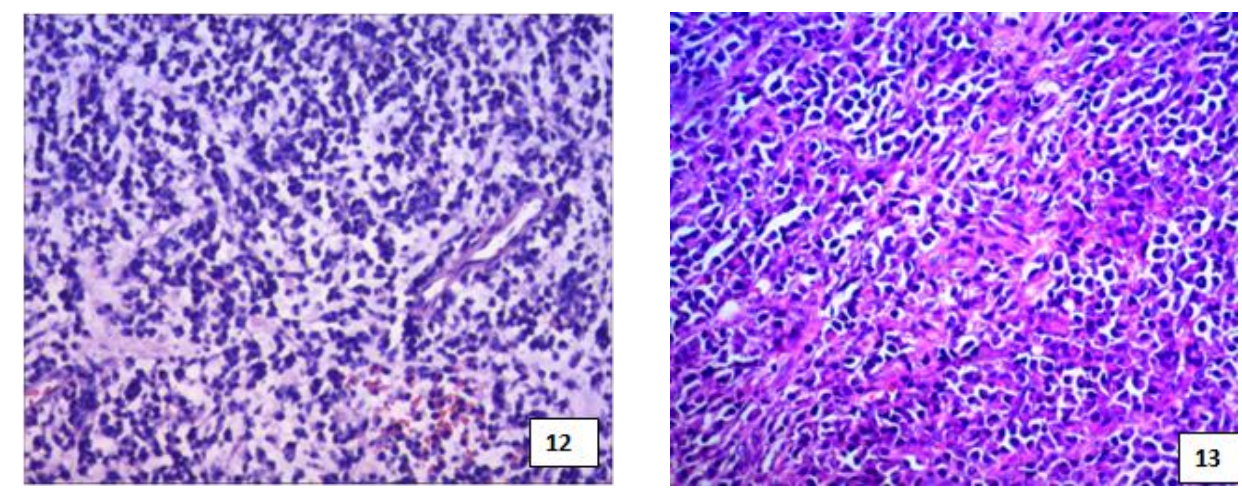

Figure12 and 13: Plasma cell tumor (skull). A typical plasma cells with typical eccentric round nuclei and prominent cytoplasm (H\&E, x200).

The most frequent sites for plasmacytoma of skull include sphenoid bone, juxtasellar region, petrous apex, cerebral convexity [6]. These locations are similar to the sites as for meningioma. In our first case, the pre-operative diagnosis was Meningioma. The postoperative histopathology report confirmed plasmacytoma. Although the negative results for serum M-protein, Bence-Jones protein and bone marrow biopsy ruled out Multiple Myeloma. It is crucial to investigate for systemic involvement for at least a year, as the solitary plasmacytoma may advance to MM later, despite negative initial presentation [2, 4]. Within a median duration of 2-4 years, more than $75 \%$ patients with apparent SBP advance to myeloma [2]. The predicted factors for development to myeloma includes tumor size $>5 \mathrm{~cm}$, old age $\geq 60$ years, high $\mathrm{M}$ protein levels, radiotherapy dose,persistence of $\mathrm{M}$ protein after treatment and spine lesions [4, 5]. However, these features are considered inconsistent and recently, the only adverse prognostic factor is concluded to be "M protein for more than 1 year after radiotherapy" [4]. 
Our second case was initially diagnosed as malignant meningioma. The biopsy showed plasmacytoma but MM couldn't be ruled out as the patient didn't follow-up with his reports. However, his SPECT-CT showed multiple lesions in his ribs confirming metastasis. The detection of multiple bony lesions (at least two hypermetabolic lesions on PET/CT) or positivity on FDG-PET scan predicts the progression of SBP to MM [2, 7]. Compared to EMP, SBP is known to have poor prognosis and higher risk of advancement to MM [2].

The imaging characteristics of SBP have also not yet been illustrated in detail. Its imaging findings are important for reducing the likelihood of misdiagnosis and for evaluating local disease and for staging. Grammatico et al, in their review article suggested that on T1-weighted images, the signal intensity is similar to muscle and on T2-weighted images, it appears hyperintense to muscle [3].

Similarly, another review article by Chargari et al. suggested hypointensity on T1-weighted images, and hyperintensity on T2-weighted images with marked homogeneous contrast enhancement [6]. However, in both our case reports, the lesion appeared isointense to the brain parenchyma in both T1WI and T2WI without any necrotic and hemorrhagic features. Several case reports published on intracranial SBP have demonstrated isointensity on both T1 and T2 weighted images with significant post-contrast enhancement [8-14]. They also demonstrated locally aggressive features like involvement and destruction of adjacent structures. Most of CT reports demonstrated hyperdensity with homogenous enhancement [7, 15-17]. These reports are in compliance with our cases.

CT displays bone destruction and lytic lesions compared to MRI. MRI is a better for evaluation of soft tissue, suspected cord or nerve root compression. DWI of solitary plasmacytoma has not been reported previously in the literature. In our first case, DWI showed no restricted diffusion. FDG-PET is the modality of choice for assessment of the skeletal abnormalities $[3,15,18,19]$.

The differential diagnosis for plasmacytoma mainly includes meningioma, metastasis, sarcoma, chordoma, eosinophilic granuloma, osteosarcoma and giant cell tumour $[8,11,20]$. Meningioma usually shows iso to hyperdensity with homogenous enhancement and a "dura tail". However, unlike plasmacytomas, meningiomas rarely cause lytic bony destruction. The involvement of dura in plasmacytoma, however, may show "dural tail" sign after contrast, but this sign remains non-specific. Osteolytic metastatic carcinoma is the commonest type of bone metastases and are usually multiple with ill defined margin, lobular appearance and surrounding edema. A metastatic lesion demonstrates heterogenous signal due to necrosis and hemorrhage and DWI shows restricted diffusion. Our first case of SBP didn’t show any restricted diffusion in DWI. The primary differential remains meningioma or metastasis.

\section{Conclusion}

The imaging features of SBP remains non-specific. More studies are required to confirm its radiological findings which can prevent misdiagnosis and invasive procedures. Hence, the recommended criterion for diagnosing and 
differentiating SBP, EMP and MM needs to be confirmed before the final diagnosis is made. Similarly, follow up at 6 weeks interval for first 6 months to measure M-protein must be performed to avoid systemic MM [21, 22]. Imaging and skeletal survey can be performed if any new bone pain or bony lesions are suspected [22].

\section{Declarations}

Human and animal rights: This is to inform that this case report does not involve the use of human subjects or experiments involving humans and animals. Hence, no harm has been done to animals and humans or provided any drugs, or caused any physical, mental or social harm.

Declaration of interests: There are no competing interests regarding this case report.

Submission declaration: This is to ensure that this case report has not been published previously.

\section{Funding}

1. The Young and Middle-aged Technical Academic Leaders Training Project in Yunnan province (Grant No. 2015HB068).

2. The Yunnan Province Medical Subject Leaders Training Project(Grant No. D-201646).

\section{References}

1. Schwartz TH, Rhiew R, Isaacson SR, et al. Association between intracranial plasmacytoma and multiple myeloma: clinicopathological outcome study. Neurosurgery 49 (2001): 1039-1044.

2. Sevil Kilciksiz, Omur Karakoyun-Celik, Fulya Yaman Agaoglu, et al. A Review for Solitary Plasmacytoma of Bone and Extramedullary Plasmacytoma. The Scientific World Journal (2012): ID 895765.

3. Grammatico S, Scalzulli E, Petrucci MT. Solitary Plasmacytoma. Mediterr J Hematol Infect Dis. 9 (2017): e2017052.

4. Soutar R, Lucraft H, Jackson G, et al. Guidelines on the diagnosis and management of solitary plasmacytoma of bone and solitary extramedullary plasmacytoma. British Journal of Haematology 124 (2004): 717-726.

5. Kumar R, Mohindro N, Radotra SD. Asian Journal of Neurosurgery, Solitary plasmacytoma of temporal bone: A rare case report 12 (2017): 95-97.

6. Chargari C, Vennarini S, Servois V, et al. Place of modern imaging modalities for solitary plasmacytoma: toward improved primary staging and treatment monitoring. Crit Rev Oncol Hematol 82 (2012):150-158.

7. Agarwal A. Neuroimaging of plasmacytoma. A pictorial review. Neuroradiol J 27 (2014): 431-437.

8. Dong L, Zhang X, Zhang H, et al. Solitary plasmacytoma of the skull: Two case reports. Oncology Letters 5 (2013): 479-482.

9. Zigouris A, Drosos D, Alexiou GA, et al. Primary plasmacytoma of the cranial vault: a case report. J Cases Journal 2 (2009): 1757-1626.

10. Azarpira N, Noshadi P, Pakbaz S, et al. Dural plasmacytoma mimicking meningioma. Turk Neurosurg 24 (2014): 403-405. 
11. Tanaka M, Shibui S, Nomura K, et al. Solitary Plasmacytoma of the Skull: a Case Report, Japanese Journal of Clinical Oncology 28 (1998): 626-630.

12. Kalwani N, Remenschneider AK, Faquin W, et al. Plasmacytoma of the Clivus Presenting as Bilateral Sixth Nerve Palsy. J Neurol Surg Rep 76 (2015): e156-159.

13. Gürbüz MS, Akmil MU, Akar E, et al. Solitary plasmocytoma of the skull. BMJ Case Rep 8 (2013).

14. Okamoto K, Ito J, Furusawa T, et al. Solitary plasmacytomas of the occipital bone: a report of two cases. Eur Radiol 7 (1997): 503-506.

15. Bakar B, Tekkok IH. Plasmocytoma of the skull vault. Turk Neurosurg 22 (2012): 95-98.

16. Nguyen, My-Linh Patel, Anand S. Plasmacytoma of the Skull. J New England Journal of Medicine (2010).

17. Manmeet G, Manisha M, Singh P. Solitary Plasmacytoma of Skull: A Rare Cytological Diagnosis. Journal of clinical and diagnostic research: JCDR (2013): 1702-1703.

18. Lu YY, Chen JH, Lin WY, et al. FDG PET or PET/CT for detecting intramedullary and extramedullary lesions in multiple Myeloma: a systematic review and meta-analysis. Clin Nucl Med 37 (2012): 833-837.

19. Fouquet G, Guidez S, Herbaux C, et al. Impact of initial FDG-PET/CT and serum-free light chain on transformation of conventionally defined solitary plasmacytoma to multiple myeloma. Clin Cancer Res 20 (2014): 3254-3260.

20. Adam C. Wilberger, Richard A. Prayson; Intracranial Involvement by Plasma Cell Neoplasms, American Journal of Clinical Pathology 146 (2016): 156-162.

21. Hu K, Yahalom J. Radiotherapy in the management of plasma cell tumors. Oncology (Williston Park) 14 (2000): 101-108.

22. Na'ara S, Amit M, Gil Z, et al. Plasmacytoma of the Skull Base: A Meta-Analysis. J Neurol Surg B Skull Base 77 (2016): 61-65.

Citation: Shrestha Sukriti, Zhu Li, Zhao Xinxiang. Imaging Findings of Solitary Plasmacytoma of the Cranium. Archives of Clinical and Medical Case Reports 2 (2018): 231-240. 